\title{
Solving Boundary-Layer Problems by Residual-Power-Series Method
}

\author{
Mohd Taib Shatnawi \\ Department of BasicSciences, Al-Huson University College, Al-Balqa’ Applied University Al-Huson 50, Jordan
}

Received: March 13, 2016 Accepted: March 29, 2016 Online Published: May 18, 2016

doi:10.5539/jmr.v8n3p68

URL: http://dx.doi.org/10.5539/jmr.v8n3p68

\begin{abstract}
In this paper, the so-called residual-power-series (RPS) method is presented for solving nonlinear boundary-layer equations. The RPS method provides a single unified treatment for the linear and nonlinear terms in the equations. The accuracy and efficiency of the RPS method is demonstrated for both a single and a system of two coupled boundary-layer equations on an unbounded domain.
\end{abstract}

Keywords: boundary-layer problem, residual-power-series, diagonal Padé approximation

\section{Introduction}

The residual-power-series (RPS) method, first proposed by (Abu Arqub, 2013), is a powerful method for solving linear and nonlinear problems. Most recently, Abu Arqub et al., (2013) employed the RPS method to solve Lane- Emden type equations. The RPS method is straightforward and simple toapply. The RPS method yields a Taylor expansion of the solution, and as a result, the exact solution is obtained whenever it is a polynomial. Moreover, the solution and all of its derivatives are applicable for each arbitrary point in a given interval. The RPS method has small computational requirements and high precision, and furthermore, it requires less time.

Many problems of interest in fluid mechanics are reduced, by introduction of suitable similarity variables, to nonlinear ordinary differential equations with appropriate boundary conditions (see for example (Vajravelu, 2001), (Kuiken, 1981), (Mishra and Mishra, 2012). The solutions of these nonlinear two-point boundary value problems are normally obtained by using, for example, the traditional finite difference methods. Approximate analytical treatments based on the Adomian decomposition method of the equations given by (Vajravelu, 2001) and (Kuiken,1981) were presented by (Kechil and Hashim, 2007a, 2007b). In this work, we shall extend for the first time the applicability of the RPS to boundary-layer equations on an unbounded domain.

\section{Residual-Power-Series (RPS) Method}

To describe the basic ideas of the RPS method (Abu Arqub, 2013) and to achieve our goal, we consider the system of initial value problem (IVP)

$$
\Psi_{i}^{(n)}(\eta)=\Gamma_{i}\left(\eta, \Psi_{i}(\eta), \Psi_{i}^{\prime}(\eta), \ldots, \Psi_{i}^{(n-1)}(\eta)\right), \quad i=1,2, \ldots, r
$$

subject to the initial conditions

$$
\Psi_{i}\left(\eta_{0}\right)=\Psi_{i, 0}, \Psi_{i}^{\prime}\left(\eta_{0}\right)=\Psi_{i, 1}, \Psi_{i}^{(n-1)}\left(\eta_{0}\right)=\Psi_{i, n-1}
$$

Where $\Gamma_{i}:\left(\eta_{0}-\varepsilon, \eta_{0}+\varepsilon\right) \times \mathcal{R}^{n} \rightarrow \mathcal{R}^{n}$ is a nonlinear analytic function, $\eta$ denotes the independent variable, $\Psi_{i}(\eta), \Psi_{i}^{\prime}(\eta), \ldots, \Psi_{i}^{(n-1)}(\eta)$ are unkown functions, and $\eta_{0}, \varepsilon$ are real.

Assume that $\mathrm{y}_{i}(\eta)$ are analytic functios on the given interval.

Therefore, these solutions can be represented as a power series as follows:

$$
\Psi_{i}(\eta)=\sum_{m=0}^{+\infty} c_{i, m}\left(\eta-\eta_{0}\right)^{m}
$$

where the coefficients $c_{i, m}$ are given by

$$
c_{i, m}=\frac{\Psi_{i}^{(m)}\left(\eta_{0}\right)}{m !}=\frac{\Psi_{i, m}}{m !}, \quad m=0,1, \ldots, n-1
$$

According to equations (2)-(4), the series solution can be written as 


$$
\Psi_{i}(\eta)=\Psi_{i, 0}+\Psi_{i, 1}\left(\eta-\eta_{0}\right)+\frac{\Psi_{i, 2}}{2 !}\left(\eta-\eta_{0}\right)^{2}+\cdots+\frac{\Psi_{i, n-1}}{(n-1) !}\left(\eta-\eta_{0}\right)^{n-1}+\sum_{m=0}^{+\infty} c_{i, m}\left(\eta-\eta_{0}\right)^{m}
$$

In practice, we approximate the solution by the $k$ th-truncated series

$$
\Psi_{i}(\eta)=\Psi_{i, 0}+\Psi_{i, 1}\left(\eta-\eta_{0}\right)+\frac{\Psi_{i, 2}}{2 !}\left(\eta-\eta_{0}\right)^{2}+\cdots+\frac{\Psi_{i, n-1}}{(n-1) !}\left(\eta-\eta_{0}\right)^{n-1}+\sum_{m=0}^{k} c_{i, m}\left(\eta-\eta_{0}\right)^{m}
$$

Now, to determine the rest of the coefficients $c_{i, m}$ for $m=n, n+1, \ldots, k$ we define $k$ th-residual function as follows

$$
R_{i}(\eta)=\Psi_{i}^{(n)}(\eta)-\Gamma_{i}\left(\eta, \Psi_{i}(\eta), \Psi_{i}^{\prime}(\eta), \ldots, \Psi_{i}^{(n-1)}(\eta)\right)
$$

It is clear that $R_{i}(\eta)=0$ for each $\eta$

$$
\sum_{m=0}^{k} c_{i, m}\left(\eta-\eta_{0}\right)^{m}
$$

Now, to determine the rest of the coefficients $c_{i, m}$ for $m=n, n+1, \ldots, k$ we define $k$ th-residual function as follows

$$
R_{i}(\eta)=\Psi_{i}^{(n)}\left(\eta_{0}\right)-\Gamma_{i}\left(\eta-\Psi_{i}(\eta), \Psi_{i}^{\prime}(\eta), \ldots, \Psi_{i}^{(n-1)}(\eta)\right)
$$

It is clear that $R_{i}(\eta)=0$ for each $\in\left(t_{0}-\varepsilon, \eta_{0}+\varepsilon\right)$, this is to confirm that these residual functions are differentiable infinitely many times at $\eta=\eta_{0}$. Moreover,

$$
\left.\frac{d^{m}}{d t^{m}} R_{i}(\eta)\right|_{\eta=\eta_{0}}=0
$$

Equation (6) and equation (10) for $m=n, n+1, \ldots, k$, generate $k-n+1$ set of linear and nonlinear algebraic equations, respectively. These equations can be easily solved by symbolic computation software such as Maple and Mathematica for the unknown coefficients $c_{i, m}$.

\section{Numerical Examples}

\subsection{Example 1}

First we consider the following nonlinear boundary-value problem (Vajravelu, 2001)

$$
\begin{gathered}
f^{\prime \prime \prime}+f f^{\prime \prime}-\frac{2 r}{r+1}\left(f^{\prime}\right)^{2}=0 \\
f(0)=0, f^{\prime}(0)=1, f^{\prime}(\infty)=0
\end{gathered}
$$

where $r$ is a real number and the primes denote differentiation with respect to $\eta$. Equations (11) and (12) model many viscous flow problems. One such example is the velocity field in the flow and heat transfer phenomenon over a nonlinearly stretching sheet (Vajravelu, 2001). We note that the special case $r=1$ admits the exact solution $f(\eta)=1-\exp (-\eta)$ with $f^{\prime \prime}(0)=-1$.

To construct the solutions of system (11)-(12) by using the RPS, we should first rewrite the boundary conditions (12) in the form of initial conditions as follows

$$
f(0)=0, f^{\prime}(0)=1, f^{\prime \prime}(0)=\alpha
$$

where $\alpha=f^{\prime \prime}(0)$ is to be determined from the boundary conditions at infinity in (12). Clearly, the first three terms of the approximation of $f(\eta)$ are $f_{0}(\eta)=0, f_{1}(\eta)=\eta$ and $f_{2}(\eta)=\frac{\alpha}{2 !} \eta^{2}$. Then the $k$ th-truncated series has the form

$$
f(\eta)=\eta+\frac{\alpha}{2 !} \eta^{2}+\sum_{m=3}^{k} c_{m}\left(\eta-\eta_{0}\right)^{m}=\frac{\alpha}{2 !} \eta^{2}+c_{3} \eta^{3}+c_{4} \eta^{4}+\cdots+c_{k} \eta^{k}
$$

Table 1. Numerical values of $\alpha$ with the corresponding values of $r$ usingdiagonal Padé approximants of $f_{32}^{\prime}$

\begin{tabular}{lcccc}
\hline$r$ & {$[5 / 5]$} & {$[7 / 7]$} & {$[10 / 10]$} & $\alpha$ of [3] \\
\hline 1 & 1.0003410482 & -0.9999999051 & -1.0000000000 & -1.0000 \\
5 & 1.1982743450 & -1.1986417560 & -1.1985277159 & -1.1945 \\
10 & 1.2365525777 & -1.2334477611 & -1.2369318045 & -1.2348 \\
\hline
\end{tabular}


To find the coefficients $c_{m}$ by our RPS algorithm, we construct the residual function as follows.

$$
\begin{aligned}
R(\eta)= & \sum_{m=3}^{k} m(m-1)(m-2) c_{m}\left(\eta-\eta_{0}\right)^{m-3}+\left(\eta+\frac{\alpha}{2 !} \eta^{2}+\sum_{m=3}^{k} c_{m}\left(\eta-\eta_{0}\right)^{m}\right) \times\left(\alpha+\sum_{m=3}^{k} m(m-\right. \\
& \left.1) c_{m}\left(\eta-\eta_{0}\right)^{m-2}\right)^{2}-\frac{2 r}{r+1}\left(1+\alpha \eta+\sum_{m=3}^{k} m c_{m}\left(\eta-\eta_{0}\right)^{m-1}\right)^{2}
\end{aligned}
$$

We have obtained the 32-term approximation to $f(\eta)$, but for the lack ofspace, only the first six terms are given below.

$$
f_{32}(\eta)=\eta+\frac{\alpha}{2} \eta^{2}+\frac{r}{3 r+3} \eta^{3}+\frac{\alpha(3 r-1)}{24(r+1)} \eta^{4}+\frac{\left[-\alpha^{2}+\left(3 \alpha^{2}+4\right) r^{2}+2\left(\alpha^{2}-2\right) r\right]}{120(r+1)^{2}} \eta^{5}+\frac{\alpha\left(19 r^{2}-18 r+3\right)}{720(r+1)^{2}} \eta^{6}+\cdots
$$

Now to determine the value of $\alpha$ we impose the condition at infinity in (12). The difficulty at infinity is overcome by employing the diagonal Padé approximants (Boyd, 1997) that approximate $f_{32}$. Table 1 shows that the values of -1 obtained via diagonal Padé approximants converge to the exact value -1 for the caser $=1$. For the cases $r=5$ and $r=10$, we make a comparison withthe results of Vajravelu, (2001)who solved the problem numerically using theintegration scheme of the fourth-order Runge-Kutta. It is observed that thenumerical results are in well agreement with that of Vajravelu, (2001). Figure 1demonstrates the agreement of the rational function Padé approximant[10/10] of $f$ and $f^{\prime}$ with the exact solution at $r=1$ and also illustrates thevariations of $f(\eta)$ and $f^{\prime}(\eta)$.

\subsection{Example 2}

Next we shall apply the RPS for solving a nonlinear system of coupled ordinary equations. Kuiken, (1981) considered the problem of cooling of a low-heat-resistance sheet that moves downwards in a viscous fluid which he modeled by the following nonlinear boundary-value problem,

$$
\begin{gathered}
f^{\prime \prime \prime}(\eta)+\theta(\eta)-\left(f^{\prime}(\eta)\right)^{2}=0 \\
\theta^{\prime \prime}(\eta)-3 \sigma f^{\prime}(\eta) \theta(\eta)=0
\end{gathered}
$$

subject to the boundary conditions

$$
\begin{gathered}
f(0)=0, f^{\prime}(0)=1, f^{\prime}(+\infty)=0 \\
\theta(0)=1, \theta(+\infty)=0
\end{gathered}
$$

where the primes denote differentiation with respect to $\eta$ and $\sigma$ is a constant.
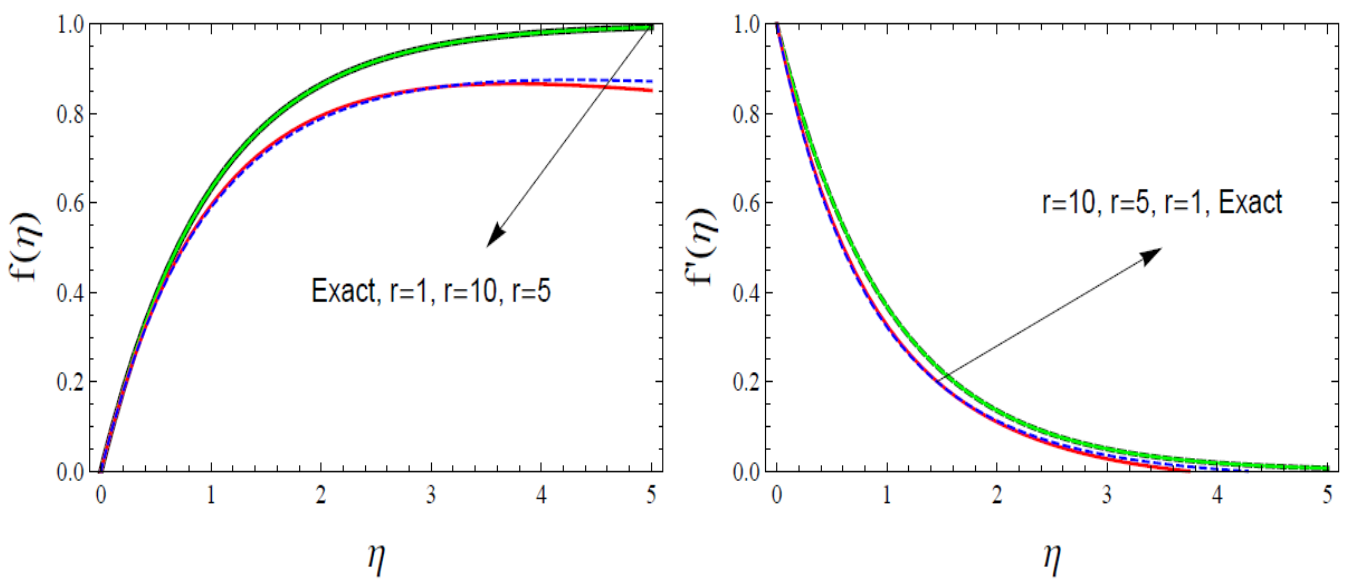

Figure 1. Variations of $f(\eta)$ and $f^{\prime}(\eta)$ using $f_{32[10 / 10]}$ and comparisonwith the exact solution for $r=1$.

To construct the solutions of system (17)-(20) by using the RPS, we takethe following initial conditions

$$
\begin{gathered}
f(0)=0, f^{\prime}(0)=1, f^{\prime \prime}(0)=\alpha \\
\theta(0)=1, \theta^{\prime}(0)=\delta
\end{gathered}
$$


where $\alpha=f^{\prime \prime}(0)$ and $\delta=\theta^{\prime}(0)$ are to be determined from the boundary conditions at infinity. Taking the first three terms of the approximation of $f(\eta)$ as $f_{0}(\eta)=0, f_{1}(\eta)=0$ and $f_{2}(\eta)=\frac{\alpha}{2 !} \eta^{2}$, then the $k$ th-truncatedseries has the form

$$
f(\eta)=\frac{\alpha}{2 !} \eta^{2}+\sum_{m=3}^{k} c_{1, m}\left(\eta-\eta_{0}\right)^{m}=\frac{\alpha}{2 !} \eta^{2}+c_{1,3} \eta^{3}+c_{1,4} \eta^{4}+\cdots+c_{1, k} \eta^{k}
$$

Next if we select the first two terms of the approximation of $\theta(\eta)$ as $\theta_{0}(\eta)=1$ and $\theta_{1}(\eta)=\delta \eta$, then the $k$ th-truncated series has the form

$$
\theta(\eta)=1+\delta \eta+\sum_{m=2}^{k} c_{2, m}\left(\eta-\eta_{0}\right)^{m}=1+\delta \eta+c_{2,2} \eta^{2}+c_{2,3} \eta^{3}+\cdots+c_{2, k} \eta^{k}
$$

To find the coefficients $c_{i, m}$ by RPS, we construct the residual functions as follows

$$
\begin{gathered}
R_{1}(\eta)=\sum_{m=3}^{k} m(m-1)(m-2) c_{1, m}\left(\eta-\eta_{0}\right)^{m-3}+1+\delta \eta+\sum_{m=2}^{k} c_{2, m}\left(\eta-\eta_{0}\right)^{m}-\left(\alpha \eta+\sum_{m=3}^{k} m c_{1, m}(\eta-\right. \\
\left.\left.\eta_{0}\right)^{m-1}\right)^{2} \\
R_{2}(\eta)=\sum_{m=2}^{k} m(m-1) c_{2, m}\left(\eta-\eta_{0}\right)^{m-2}-3 \sigma\left(\alpha \eta+\sum_{m=3}^{k} m c_{1, m}\left(\eta-\eta_{0}\right)^{m-1}\right) \times\left(1+\delta \eta+\sum_{m=2}^{k} c_{2, m}\left(\eta-\eta_{0}\right)^{m}\right)
\end{gathered}
$$

We have obtained the 23th-order and 22th-order approximations to $f(\eta)$ and $\theta(\eta)$, respectively, but for the lack of space, only the first five terms produced below.

$f_{23}(\eta)=$

$\frac{\alpha}{2} \eta^{2}-\frac{1}{6} \eta^{3}-\frac{\beta}{24} \eta^{4}+\frac{\alpha^{2}}{60} \eta^{5}+\frac{1}{240}(-\alpha \sigma-2 \sigma) \eta^{6}+\frac{(-6 \alpha \beta \sigma-8 \alpha \beta+3 \sigma+6)}{5040} \eta^{7}+\frac{\left(5 \alpha^{3}+3 \beta \sigma+5 \beta\right)}{10080} \eta^{8}+$

$\frac{\left(-18 \alpha^{2} \sigma^{2}-21 \alpha^{2} \sigma-66 \alpha^{2}+6 \beta^{2} \sigma+10 \beta^{2}\right)}{181440} \eta^{9}-\frac{\alpha\left(15 \alpha \beta \sigma^{2}+19 \alpha \beta \sigma+42 \alpha \beta-24 \sigma^{2}-31 \sigma-56\right)}{604800} \eta^{10}+\cdots$

$\theta_{22}(\eta)=$

$1+\beta \eta+\frac{\alpha}{2} \eta^{3}+\frac{\sigma(2 \alpha \beta-1)}{8} \eta^{4}-\frac{\beta \sigma}{10} \eta^{5}+\frac{\sigma\left(6 \alpha^{2} \sigma+\alpha^{2}-2 \beta^{2}\right)}{120} \eta^{6}+\frac{\alpha \sigma(15 \alpha \beta \sigma+5 \alpha \beta-24 \sigma-3)}{840} \eta^{7}-\frac{\sigma(123 \alpha \beta \sigma+22 \alpha \beta-24 \sigma-3)}{6720} \eta^{8}+$

$\frac{\sigma\left(126 \alpha^{3} \sigma^{2}+126 \alpha^{3} \sigma+10 \alpha^{3}-168 \alpha \beta^{2} \sigma-28 \alpha \beta^{2}+195 \beta \sigma+31 \beta\right)}{60480} \eta^{9}+\cdots$

The undetermined values of $\alpha$ and $\beta$ are calculated from the boundary onditions at infinity in (19) and (20). The results presented in Tables 2 and 3 are in good agreement with that given by (Kuiken, 1981). Figures 2-3 illustrate the variation of $f(\eta), f(\eta)$ and $\theta(\eta)$ approximated by the diagonal Padéapproximants in the cases $\sigma=0.1, \sigma=1$ and $\sigma=10$.

Table 2. Numerical values of $\alpha$ usingdiagonal Padé approximants of $f_{23}^{\prime}$ and $\theta_{22}$.

\begin{tabular}{lcccc}
\hline$\sigma$ & {$[4 / 4]$} & {$[5 / 5]$} & {$[6 / 6]$} & $\alpha$ of $[4]$ \\
\hline 0.001 & 1.1135529418 & 1.1272760416 & 1.1252849854 & 1.1231381347 \\
0.01 & 1.0631737963 & 1.0741895683 & 1.0638385351 & 1.0633808585 \\
0.1 & 0.9128082210 & 0.9238226280 & 0.9242158493 & 0.9240830397 \\
1 & 0.6941230861 & 0.6998750497 & 0.6932195158 & 0.6932116298 \\
10 & 0.4511240728 & 0.4502429544 & 0.4476712316 & 0.4471165250 \\
100 & 0.2679197151 & 0.2681474363 & 0.2641295627 & 0.2645235434 \\
1000 & 0.2204061432 & 0.1524783266 & 0.1500456755 & 0.1512901971 \\
10000 & 0.0858587180 & 0.0858519249 & 0.0844775473 & 0.0855408524 \\
\hline
\end{tabular}


Table 3. Numerical values of $\alpha$ usingdiagonal Padé approximants of $f_{23}^{\prime}$ and $\theta_{22}$.

\begin{tabular}{lcccc}
\hline$\sigma$ & {$[4 / 4]$} & {$[5 / 5]$} & {$[6 / 6]$} & $\alpha$ of $[4]$ \\
\hline 0.001 & -0.0371141028 & -0.0415417739 & -0.0436188230 & -0.0468074648 \\
0.01 & -0.1274922800 & -0.1221616907 & -0.1351353865 & -0.1357607439 \\
0.1 & -0.3621215470 & -0.3505589981 & -0.3499273453 & -0.3500596733 \\
1 & -0.7694165843 & -0.7614765813 & -0.7698955992 & -0.7698611967 \\
10 & -1.5028543431 & -1.5007437650 & -1.4985484075 & -1.4970992078 \\
100 & -2.7627624234 & -2.7637067330 & -2.7445541894 & -2.7468855016 \\
1000 & -5.7787858408 & -4.9468469883 & -4.9104728566 & -4.9349476252 \\
10000 & -8.8057265644 & -8.8032691004 & -8.7384279086 & -8.8044492660 \\
\hline
\end{tabular}
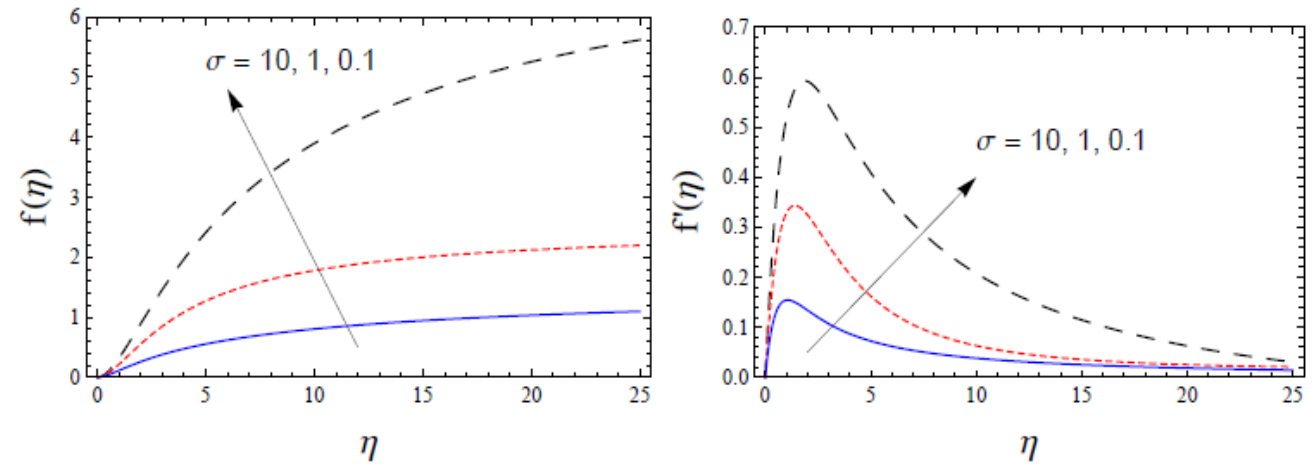

Figure 2. Variations of $f(\eta)$ and $f^{\prime}(\eta)$ using $f_{23[6,6]}$ for $\sigma=0.1$, $\alpha=0.9242158493$ and $\beta=-0.3499273453 . f_{23[5,5]}$ for $\sigma=1, \alpha=0.6941230861$ and $\beta=-0.7694165843 . f_{23[4,4]}$ for $\sigma=10, \alpha=0.4476712316$ and $\beta=-1.4985484075$.

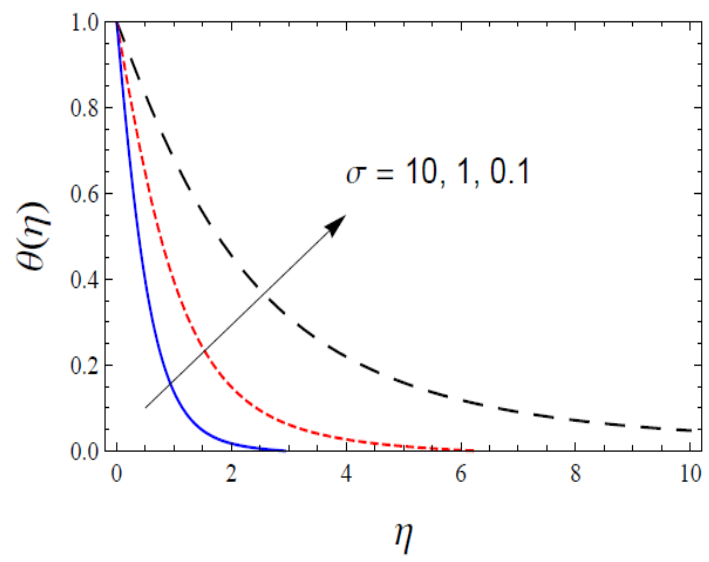

Figure 3. Variations of $\theta(\eta)$ and $\theta_{22[6,6]}$ for $\sigma=0.1, \alpha=0.9242158493$ and $\beta=-0.3499273453$. $\theta_{22[5,5]}$ for $\sigma=1, \alpha=0.6932195158$ and $\beta-0.7698955992 . \theta_{22[4,4]}$ for $\sigma=10, \alpha=0.4476712316$ and $\beta=$ -1.4985484075 .

\section{Conclusion}

The residual-power-series method was employed to solve nonlinear boundary-value problems. The RPS combined with Padé approximants are also shown to be a promising tool in solving two-point boundary value problems consisting of systems of nonlinear differential equations. The RPS method provides a single unified treatment for the linear and 
nonlinear termsin the equations. The accuracy and efficiency of the RPS method isdemonstrated for both a single and a system of two coupled boundary-layerequations on an unbounded domain.

\section{Acknowledgments}

The author acknowledges with gratitude thevaluable comments and suggestions of the editor and the referees, which helped in revising the manuscript.

\section{References}

Abu Arqub, O. (2013). Series solution of fuzzy differential equations under strongly generalized differentiability. J. Adv. Res. Appl. Math, 5, 31-52. http://dx.doi.org/10.5373/jaram.1447.051912

Abu Arqub, O., El-Ajou, A., Bataineh, A. Sami, \& Hashim, I. (2013). A representation of the exact solution of generalized Lane-Emden equations using a new analytical method. Abstract and Applied Analysis, 2013, 1-10. http://dx.doi.org/10.1155/2013/378593

Boyd, J. P. (1997). Padé approximant algorithm for solving nonlinear ordinary differential equation boundary value problems on an unbounded domain. Comput. Phys, 11, 299-303. http://dx.doi.org/10.1063/1.168606

Kechil, S. Awang., Hashim I., \& Jiet S. (2007a). Approximate analytical solutions for a class of laminar boundary-layer equations. Chinese Physics Letters, 24, 1981-1984. http://dx.doi.org/10.1088/0256-307X/24/7/054

Kechil, S. Awang., \& Hashim I. (2007b). Non-perturbative solution of free-convective boundary-layer equation by Adomian decomposition method, Phys. Lett. A, 363, 110-114. http://dx.doi.org/10.1016/j.physleta.2006.11.054

Kuiken, H. K. (1981). A 'backward' free-convective boundary layer. Quarterly J. Mech. Appl. Math. 34, $397-413$. http://dx.doi.org/10.1093/qjmam/34.3.397

Mishra, V.N., \& Mishra L.N. (2012). Trigonometric Approximations of signals (Functions) in $\mathrm{L}_{\mathrm{p}}$-norm. International Journal of Contemporary Mathematical Sciences, 7, 909-918.

Vajravelu, K. (2001). Viscous flow over a nonlinearly stretching sheet. Appl. Math. Computat. 124, $281-288$. http://dx.doi.org/10.1016/S0096-3003(00)00062-X

\section{Copyrights}

Copyright for this article is retained by the author(s), with first publication rights granted to the journal.

This is an open-access article distributed under the terms and conditions of the Creative Commons Attribution license (http://creativecommons.org/licenses/by/3.0/). 